\title{
Enquête
}

Archives de la revue Enquête

5 | 1997

Débats et controverses

\section{Controverses et styles de raisonnement}

Débats sur la statistique de population au XIX ${ }^{\mathrm{e}}$ siècle en France et en Angleterre

Controversies and Styles of Reasoning. Debates over Population Statistics in 19th century France and England

\section{Libby Schweber}

Traducteur : Morgane Labbé

\section{(2) OpenEdition}

12 Journals

Édition électronique

URL : http://journals.openedition.org/enquete/1113

DOI : 10.4000/enquete. 1113

ISSN : 1953-809X

Éditeur :

Cercom, Éditions Parenthèses

Édition imprimée

Date de publication : 1 septembre 1997

Pagination : 83-108

Référence électronique

Libby Schweber, "Controverses et styles de raisonnement », Enquête [En ligne], 5 | 1997, mis en ligne le 15 juillet 2013, consulté le 21 mars 2020. URL : http://journals.openedition.org/enquete/1113 ; DOI : https://doi.org/10.4000/enquete.1113 


\title{
Controverses et styles de raisonnement
}

\author{
Débats sur la statistique de population au XIX ${ }^{\mathrm{e}}$ siècle en France et en \\ Angleterre
}

Controversies and Styles of Reasoning. Debates over Population Statistics in 19th century France and England

\section{Libby Schweber}

Traduction : Morgane Labbé

1 Depuis dix ans, une abondante littérature sur l'histoire de la statistique au xIx siècle a enrichi nos connaissances sur les relations étroites qui lient les différents usages de la statistique, comme outils de représentation, d'explication et d'administrationintervention ${ }^{1}$. Les rares travaux qui comparent des expériences nationales ne font que les juxtaposer et ne se servent pas de leur comparaison pour construire leur objet et mieux comprendre les cas individuels. La « controverse » semble une source historique particulièrement efficace pour étudier des variations nationales entre formes de raisonnements statistiques; ainsi en est-il des débats qui ont porté sur des questions techniques dans le domaine de la statistique de population, en France et en Angleterre, dans les années 1830-1850. Les questions analytiques qui se posent à leur propos, et leur contribution à l'enquête historique, concernent les modes d'argumentation sollicités, les styles de raisonnement qu'ils révèlent et leurs relations mutuelles.

2 L'expression «styles de raisonnement » a été introduite par Ian Hacking, qui les définit comme « the ways in which we know, find out and evolve skills of thinking, asking and investigating ${ }^{2}$ ». Dans sa conception, l'introduction d'un nouveau style de raisonnement est étroitement liée à celle de nouvelles entités, de nouvelles explications, de nouveaux critères épistémologiques et de nouvelles techniques de preuve. Dans sa discussion des styles de raisonnement statistiques, Hacking identifie trois formes successives: la description, le raisonnement inférentiel - visant à l'identification des causes et ainsi à la formation des politiques - et la modélisation mathématique. Alors que le premier est ancien, les deux derniers furent successivement introduits au cours $d u$ XIX et au début 
$\mathrm{du} \mathrm{Xx}^{\mathrm{e}}$ siècle. Comme Hacking le souligne, leur introduction successive fut le résultat d'un long processus, marqué par différents types de micro-événements dans des domaines divers.

En comparant le modèle occidental et libéral du raisonnement statistique avec ses développements en Europe orientale, aussi bien T. Porter que I. Hacking ont mis en évidence des différences entre, d'un côté, la France et l'Angleterre, où s'est développée et diffusée l'approche probabiliste - associée à l'utilisation des nombres pour identifier des lois et des causalités -, et, de l'autre côté, l'Allemagne et d'autres pays d'Europe orientale où s'est maintenue une forme plus descriptive de la pensée statistique. Ils traitent ainsi la France et l'Angleterre comme un seul et même cas.

La recherche que j'ai menée m'a au contraire révélé d'importantes différences (dans les modes nationaux d'argumentation comme dans les styles de raisonnement statistique) entre ces deux pays. Il est possible de les explorer à partir de deux controverses sur l'usage des tables de mortalité comme outil d'enquête sociale. La première est constituée par un échange de lettres adressées à l'Académie des Sciences entre 1837 et 1838 et publiées dans les Comptes Rendus de l'Académie, la seconde par une série d'articles publiés dans le Journal de la Société de Statistique de Londres au cours de l'année 1844.

5 Si deux cas ne suffisent évidemment pas pour établir une thèse générale sur les différences nationales, ils permettent toutefois de montrer comment ils s'insèrent dans un type de problématique historique et méthodologique précis, dont on exposera les hypothèses initiales. L'étude maintenant classique de Donald MacKenzie sur la controverse Pearson/Yule, qui eut lieu au tout début du siècle à propos des "coefficients de corrélation ${ }^{3}$ ", en constitue le cadre d'analyse. Les conclusions concernant le cas français prendront aussi en compte l'analyse que l'on a faite du débat sur la dépopulation qui se déroula en 1867 à l'Académie de Médecine, et qui, mettant également en jeu des différences techniques sur l'usage des statistiques, présente beaucoup de similarités avec le débat de $1837^{4}$.

6 Une comparaison de ces quatre débats fait apparaître des différences nationales frappantes dans la manière dont les auteurs combinent des considérations techniques, professionnelles, politiques et idéologiques. Dans les deux débats anglais, celui de 1844 et celui de 1900 décrit par MacKenzie, ces différents types de considérations se trouvent complètement intégrés. Des considérations techniques fournissent une "proxy " pour des considérations politiques et vice versa. Cette articulation contribue alors au développement de techniques et pratiques de mesure nouvelles.

7 En France, en revanche, les débats de 1837 et de 1867 furent caractérisés par une disjonction entre les différents types d'arguments. Les protagonistes n'associent pas de manière systématique les considérations de divers ordres. En d'autres termes, les positions des individus sur des questions techniques ne permettent pas de prévoir leurs positions sur des questions politiques et professionnelles.

De manière similaire, à un niveau rhétorique, les différents types d'arguments ne sont pas utilisés pour en imposer d'autres. Finalement, ces débats ne semblent pas avoir influencé de manière significative l'état de la pratique statistique ; ils n'ont contribué ni à l'introduction d'innovations techniques, ni à l'introduction de nouveaux critères épistémologiques, alors que ce fut le cas en Angleterre. Tandis que les statisticiens anglais ont adopté un style de raisonnement inférentiel dès les années 1840, ce style de 
raisonnement n'a pas été introduit en France chez les statisticiens (à la différence des mathématiciens) avant le $\mathrm{xx}^{\mathrm{e}}$ siècle.

\section{Les « controverses » dans la sociologie et l'histoire des sciences}

\section{Un objet d'enquête privilégié}

9 En sociologie des sciences, l'étude de la controverse comme objet d'enquête est généralement associée à une approche particulière, d'abord développée à Edimbourg et à Bath sous le titre de Sociology of Scientific Knowledge (SSK). Appelée école " constructiviste » ou " constructiviste/relativiste ${ }^{5}$ », la SSK se développa à la fois en opposition aux interprétations philosophiques de l'histoire des sciences - qui expliquent le contenu de la connaissance scientifique par des considérations de pure cohérence rationnelle et par des procédures de validation expérimentale - et à l'école institutionnaliste telle qu'elle fut représentée par Robert Merton et Joseph Ben-David. Alors que les institutionnalistes concentraient leur enquête sur les conditions sociales et institutionnelles qui favorisaient le progrès de la connaissance scientifique - laissant la question du contenu aux philosophes -, la SSK soulignait la nécessité d'une analyse sociologique du contenu des idées scientifiques. Au cours du XIX siècle, ce double intérêt - discréditer les positions philosophiques et développer une sociologie du contenu des idées scientifiques - conduisit la SSK à privilégier les controverses comme objet de l'enquête empirique.

10 S'appuyant sur Wittgenstein, des théoriciens de la SSK comme Barny Barnes ${ }^{6}$ conceptualisèrent la science comme une suite de revendications de savoir (knowledge claims) situées dans un réseau conceptuel. La production du savoir implique l'élaboration d'un réseau particulier ou une série d'associations. Dans ce cadre, la question de la croyance scientifique devient celle de l'explication du consensus sur le statut épistémologique (facticity) d'une revendication particulière. Ainsi, alors que les institutionnalistes expliquaient la croyance scientifique par les techniques de démonstration et de preuve (comme la validation expérimentale) et s'interrogeaient sur le rôle des organisations formelles et des mécanismes institutionnels ayant facilité ce consensus, la SSK problématise la possibilité même du consensus. Une grande partie du travail de la SSK au cours de cette période se compose d'études empiriques destinées à identifier les différents éléments « extra-scientifiques » en jeu dans la réalisation du consensus, pour une revendication scientifique particulière.

11 Les présupposés de la SSK selon lesquels les procédures scientifiques standard n'établissent pas à elles seules le savoir et que d'autres facteurs sont nécessaires pour expliquer le consensus, les conduisent à privilégier la controverse comme objet d'enquête $^{7}$. Les controverses fournissent, d'une part, un objet parfait pour les constructivistes qui veulent démontrer le caractère non conclusif des procédures scientifiques formelles. D'autre part, elles apportent un aperçu sur l'hétérogénéité des facteurs en jeu dans la production des connaissances. Comme Bruno Latour l'explique, les controverses fournissent un éclaircissement (account) qui diffère radicalement de la littérature scientifique qui se présente formellement comme telle ${ }^{8}$.

12 Si l'on passe en revue la multiplicité des études sur les controverses produites au cours des dernières années de la SSK, on peut identifier deux types de recherches : d'une part 
des études de "clôture " visant à montrer l'incapacité des techniques scientifiques traditionnelles à résoudre des débats et d'autre part des études de croyances, qui cherchent à expliquer pourquoi des individus adoptent une position plutôt qu'une autre au cours du débat. En général, l'intérêt de la SSK à discréditer les théories logicopositivistes de la science et ses engagements de principe dans des formes contingentes d'explication découragea ses adeptes de toute forme de généralisation. Dans la mesure où ils le généralisèrent, ce style de travail a tendu à revêtir la forme de typologies ou de classifications portant sur les considérations impliquées dans la controverse scientifique. Dans la plupart des cas, ces listes ont été construites en opposition au modèle qui privilégiait l'exercice de la raison et l'expérimentation. Leurs classifications juxtaposèrent ainsi des considérations «scientifiques" et "techniques" avec des considérations « extra-scientifiques ${ }^{9} »$.

\section{Une critique de l'explication « par intérêt »}

13 Au sein de la SSK deux types d'objections ont été soulevées contre cette première approche des controverses. L'attaque la plus directe vint de Steven Woolgar, un des principaux adeptes de l'analyse du discours, qui critiqua des auteurs comme Steven Shapin et Donald MacKenzie pour la priorité ontologique qu'ils attribuaient à la notion d'«intérêt». Selon lui, beaucoup d'études de controverses font l'hypothèse que les revendications scientifiques sont le produit d'une construction en cours, alors que les « intérêts » sont donnés ${ }^{10}$.

Une seconde critique, exprimée plus tard, porte sur la concentration de la SSK sur des réseaux de concepts, au dépend de la variété des autres formes de pratique. Comme Pickering l'explique, la notion d'intérêt fut initialement introduite pour expliquer l'extension des réseaux conceptuels dans une direction plutôt qu'une autre ${ }^{11}$. Si cette approche a effectivement mis en évidence le rôle des facteurs sociaux dans la production du savoir scientifique, elle obscurcit « la richesse de la science en train de se faire, le travail dense de construction des instruments, de planification, de gestion et d'interprétation des expériences, de l'élaboration des théories, de la négociation avec la gestion du laboratoire, les revues et les agences de financement, et ainsi de suite ». À la place des explications de clôture basées sur l'intérêt, des approches plus récentes soulignent la multiplicité et l'hétérogénéité des types de mécanismes à l'œuvre ${ }^{12}$.

Plus généralement, l'intérêt croissant pour les aspects non cognitifs de la production du savoir scientifique a été accompagné par un abandon des controverses en tant qu'objet explicite d'enquête. Depuis le milieu des années quatre-vingt l'étude des controverses s'est « routinisée ». C'est pourquoi, alors que les controverses continuent à figurer dans le répertoire de la SSK, elles ont en grande partie cessé d'être un objet explicite de réflexion. La quasi-absence de discussions théoriques et méthodologiques, comme le grand nombre d'études empiriques qui continuent à être produites, témoignent de ce déplacement.

L'approche centrée sur les « intérêts » de la controverse peut être aussi mise en défaut par deux autres raisons, l'une mineure, l'autre plus importante. La première concerne les typologies que les théoriciens de "l'intérêt» utilisent pour caractériser les différentes considérations en jeu dans un débat particulier. Comme on l'a mentionné auparavant, ces études mettent souvent en contraste les intérêts «scientifiques » ou «techniques » et «extra-scientifiques ». Si ces catégories produisent des descriptions 
qui sont intuitivement accessibles, elles ne sont pas toujours conformes aux catégories des acteurs. Le danger est qu'elles imposent des délimitations contemporaines entre science et non-science ou entre considérations techniques et non techniques sur les données empiriques, et qu'en procédant ainsi, elles dissimulent les axes et les tensions autour desquelles une controverse particulière s'est développée dans les faits.

Un second problème plus sérieux concerne la quasi-absence de considérations institutionnelles dans la plupart des études de la SSK. Comme on l'a signalé, la SSK s'était à l'origine développée en opposition à la fois à l'approche "institutionnaliste " américaine et à l'image particulière du " positivisme logique ». Une des conséquences de cette prise de position fut l'exclusion des considérations institutionnelles des modèles explicatifs de la SSK. Les études sur les controverses, par exemple, passèrent directement des intérêts techniques aux intérêts idéologiques et sociaux, négligeant l'influence des facteurs comme les organisations formelles, les structures des réseaux sociaux et même les frontières disciplinaires et épistémologiques institutionnalisées qui règlent la production et la circulation des revendications scientifiques.

Cette négligence, tout comme le but polémique d'un grand nombre de ces premières études, conduisit à un curieux paradoxe. D'une part, l'étude des controverses devint un point de passage presque indispensable dans l'étude des revendications scientifiques. D'autre part, peu de tentatives furent entreprises pour expliquer les variations dans la conduite des controverses. Les controverses furent tout simplement utilisées comme une ouverture pour observer les « intérêts » extrascientifiques.

Dans la discussion qui suit, nous proposons une utilisation alternative de la controverse comme type de source historique centrée sur la manière dont des considérations différentes s'articulent à l'intérieur d'un débat en même temps que sur les configurations institutionnelles qui les sous-tendent.

\section{L'Académie des Sciences de Paris, 1838}

\section{Le débat}

Le débat de 1837 débute quand Moreau de Jonnès, alors directeur de la Statistique Générale de la France (SGF) récemment créée, adresse une lettre à l'Académie des Sciences pour demander que le Prix Montyon ${ }^{13}$ ne soit pas décerné à M. Demonferrand, auteur d'un des nombreux travaux soumis au concours annuel. La lettre de Moreau de Jonnès était inhabituelle par le fait qu'elle consistait en une tentative de la part d'une personne étrangère au concours d'influencer la décision du jury. Au lieu d'ignorer la requête, l'Académie publia la lettre dans les actes hebdomadaires et Demonferrand répliqua, inaugurant ainsi un échange de lettres entre les deux hommes qui se poursuivit tout au long de l'année ${ }^{14}$.

Pour comprendre pourquoi Moreau de Jonnès fut si préoccupé par l'essai de Demonferrand, il est utile d'examiner les circonstances qui conduisirent à sa production. En 1836, Moreau de Jonnès avait publié le premier volume d'une série qui devait en comprendre quatorze, sur l'état de la France ${ }^{15}$. En 1837, l'Académie des Sciences demanda à M. Demonferrand, alors membre correspondant, d'évaluer, en présence des faits, cette publication. Pour tester la qualité des données, Demonferrand utilise les données sur la conscription et les statistiques départementales sur le mouvement de la population. Il joint à son rapport sur «le degré de probabilité des 
documents et des résultats », un calcul d'estimation de l'erreur au niveau national et départemental. Cette analyse lui fournit des données pour construire une nouvelle table de mortalité de la France qu'il soumet sous le titre Essai sur les lois de la mortalité et de la population en France. En plus de ces tables, l'essai de Demonferrand comprenait une discussion des causes possibles de diverses observations, et notamment, des différences entre la mortalité des hommes et des femmes.

L'essai constituait ainsi une évaluation de l'appareil administratif au niveau local et national et une contribution à l'étude des lois de populations. L'échange de lettres entre Moreau de Jonnès et Demonferrand invoquait des considérations techniques, épistémologiques et politiques. Un examen des arguments donne un aperçu de la manière dont ces types de considérations étaient articulés dans une confrontation entre deux modes de raisonnement statistique. Alors que Moreau de Jonnès revendique un modèle descriptif, Demonferrand conçoit ses tables comme des représentations de « lois de la mortalité ».

Dans sa première lettre, Moreau de Jonnès critiquait la préférence de Demonferrand pour les données préfectorales sur celles "socialement parfaites" de la SGF, et lui reprochait même sa tentative de construire une table de mortalité. Selon lui, la qualité des statistiques dépendait d'abord et avant tout de la qualité et de l'exhaustivité des données plutôt que des compétences du calculateur. Or, plus de la moitié des sept tables de mortalité existantes s'étaient appuyées sur moins d'un trois millième de la population de la France. Aucun statisticien digne de ce nom, avançait Moreau de Jonnès, n'oserait revendiquer de telles pratiques.

24 Cette critique des données de Demonferrand renvoie à la position épistémologique plus générale de Moreau de Jonnès concernant la base du savoir statistique. Dans son traité de 1847 intitulé Éléments de Statistique, il rejette explicitement les tables de mortalité comme de simples «fictions ». Sa position repose sur la conviction que la certitude du savoir statistique dépend d'une correspondance une à une entre les unités physiques et les nombres « historiques » ou « vrais ».

Dans une lettre ultérieure, Moreau de Jonnès introduit un troisième type de critique concernant l'usage public de telles tables et la responsabilité de l'Académie si elle intégrait de telles considérations dans ses décisions scientifiques, en l'occurrence l'attribution du Prix Montyon. En janvier 1838, Moreau de Jonnès explique qu'il y a 27 compagnies d'assurances en France, dont plus de la moitié ont été fondées au cours de l'année précédente. Parmi celles-ci, les deux-tiers sont spécialisées dans les assurances sur la vie: leurs contrats se fondent sur des tables de mortalité particulières et la confiance publique est directement proportionnelle à l'autorité scientifique de ces tables. Que l'Académie des Sciences attribue le prix à Demonferrand, déclare Moreau de Jonnès, et elle sanctionnerait de son autorité sa table, la plaçant ainsi au-dessus de celles déjà utilisées, et minant la confiance du public envers les compagnies d'assurance.

$26 \mathrm{Au}$ lieu de défendre la qualité de ses données, Demonferrand répond à Moreau de Jonnès en invoquant un autre modèle épistémologique. Selon lui, le savoir scientifique est par nature très approximatif. La question de la science, avance-t-il, n'est pas de savoir si les données sont fiables, mais plutôt d'évaluer le degré d'approximation requis : «Malgré les imperfections reconnues des tableaux, avec quelle approximation pouvait-on, par une discussion approfondie, en tirer les lois de la mortalité ? Telle est la question que je me suis proposée de résoudre, telle me semble être la marche 
philosophique des sciences. » Demonferrand conclut alors sa discussion en réaffirmant les fondements scientifiques de sa tentative de statuer sur le savoir: "Je n'ai jamais espéré pouvoir tirer des documents actuels une plus grande approximation et je la crois supérieure à celle de tous les travaux antérieurs sur le même sujet ${ }^{16}$.» Pour Demonferrand, la décision de l'Académie ne pourrait en aucune façon influencer la viabilité économique de ces nouvelles compagnies.

\section{L'articulation des axes et des modes de raisonnement}

Comme cette discussion l'indique, la controverse entre Moreau de Jonnès et Demonferrand fait intervenir des différences techniques sur le choix des données, des questions épistémologiques concernant la nature du savoir scientifique et des différents types d'énoncés, et des questions politiques/institutionnelles concernant les conséquences publiques de la sanction d'une table de mortalité particulière et la responsabilité propre de l'Académie. De ces trois questions, seules les deux premières semblent avoir été étroitement articulées. À la différence du débat Pearson/Yule où les intérêts techniques, cognitifs, idéologiques et sociaux se renforçaient mutuellement, les questions "extra-scientifiques " semblent dans le cas du débat Moreau de Jonnès/ Demonferrand avoir eu peu d'influence sur sa dynamique. L'argument de Moreau de Jonnès à propos des compagnies d'assurance s'appuie sur les conséquences d'un changement dans les contrats, et non sur la valeur des données ou des problèmes d'épistémologie. N'étant engagé dans aucune compagnie d'assurance, avant comme après le débat, les intérêts commerciaux de celles-ci ne pouvaient pas davantage être considérés comme des raisons expliquant ou même informant ses positions épistémologiques.

Dans la mesure où des considérations institutionnelles étaient en jeu, elles semblent avoir impliqué la reconnaissance des données de la SGF comme lieu légitime de la production scientifique. Dans ce contexte il est peut-être important de noter que les travaux statistiques basés sur les données de la SGF étaient systématiquement rejetés par l'Académie des Sciences sous prétexte que leurs auteurs manquaient de savoir de première main sur leurs données.

Ce débat peut être ainsi considéré comme l'un de ces nombreux « micro-événements » qui contribuent à l'introduction d'un nouveau style de raisonnement statistique. La position épistémologique de Demonferrand contraste avec le modèle descriptif adopté par Moreau de Jonnès qui repose sur la réduction des statistiques aux nombres absolus. De même, la suggestion de Demonferrand d'utiliser les tables de mortalité pour distinguer entre les différentes causes de mortalité ouvre la voie à ce que Hacking décrit comme un usage inférentiel des statistiques, quand bien même Demonferrand lui-même ne va pas au-delà de la mention de cet usage. Or le jury du Prix Montyon a reformulé à sa place les termes du débat.

En 1838, la Commission de l'Académie des Sciences décide de ne pas décerner le prix à Demonferrand. Si cette décision peut apparaître comme la victoire des arguments épistémologiques et politiques de Moreau de Jonnès, le rapport du jury et des événements ultérieurs suggèrent une autre lecture. Au lieu de rejeter entièrement son texte, la commission demande à Demonferrand de soumettre à nouveau son essai l'année suivante, avec une discussion sur « la marche qu'il a suivie » et les raisons de 
chaque étape. C'est sur la base de ce texte allongé que Demonferrand reçoit le Prix Montyon pour 1838.

Dans le rapport de 1838, les membres de la Commission justifient leur décision. Ils font d'abord la liste de tous les types de résultats empiriques - le nouveau savoir - que comporte l'essai. Puis ils insistent sur les qualités personnelles de l'auteur de l'essai, et sur l'importance $d u$ travail investi. "On sent combien d'aussi nombreuses déterminations ont dû coûter de soins et de peines, concluent-ils, lorsqu'on pense que M. Demonferrand a basé ses calculs sur des nombres de décès incomparablement plus grands que ceux dont les savants, qui se sont livrés aux mêmes recherches, ont fait usage. » Finalement les membres du jury du Prix Montyon excluent de leur évaluation les calculs de Demonferrand, qu'ils ne considèrent pas comme pertinents pour l'attribution du prix : «Il n'entrait point dans les devoirs de la Commission de vérifier les calculs; elle a dû se borner à reconnaître la bonté des méthodes qui ont dirigé l'auteur dans le choix et la classification des matériaux [...]. Un aussi vaste travail, qui a demandé tant de recherches et des calculs si multipliés, remplit parfaitement l'intention du fondateur du prix qui fut d'avancer la statistique de la France ${ }^{17}$.»

Ce dernier commentaire sur la responsabilité de la Commission dans la vérification des calculs de Demonferrand peut être interprété de deux manières. Soit il renvoie au contrôle des erreurs de routine sur l'addition et la division, et ainsi à la pratique de la statistique descriptive, soit il se réfère aux calculs mathématiques de Demonferrand concernant le niveau de l'erreur ${ }^{18}$. Dans un cas, il situe l'art de la statistique dans la production laborieuse des données plutôt que dans le travail de calcul, dans l'autre, il traduit l'intérêt porté par l'Académie à son monopole sur les mathématiques ainsi qu'à l'établissement d'une frontière claire avec les pratiques moins prestigieuses de la statistique ${ }^{19}$.

Le rapport de la Commission signale ainsi l'existence d'un troisième modèle pour la statistique, légèrement différent de celui de Moreau de Jonnès et loin des positions épistémologiques de Demonferrand. Alors que Demonferrand présente ses tables de mortalité comme une contribution à une science qui est approximative et abstraite et dont l'autorité repose sur l'usage des mathématiques pour le contrôle de l'erreur (le concept de probabilité du xvIII siècle), l'Académie décerne ses prix pour la qualité des données recueillies et pour les nouveaux faits mis en évidence par Demonferrand. Et tandis que Moreau de Jonnès soutient un modèle descriptif de statistique (dans lequel l'autorité du savoir statistique dépend de sa correspondance parfaite avec les observations et de l'exhaustivité des données nationales produites par la SGF), l'Académie récompense un second modèle descriptif dont l'autorité repose sur les qualités personnelles du chercheur et, en conséquence, sur le caractère local des données. En termes de clôture du débat, le rôle institutionnel de l'Académie comme arbitre suprême conduit à une reformulation du débat en une controverse sur deux types de données, plutôt que sur deux types de propositions ou de modèles épistémologiques et, au final, à une décision en faveur du recueil des données à l'échelle locale.

Deux traits de ce débat semblent ainsi importants. Le premier concerne la très faible articulation entre les différentes considérations en jeu dans le débat et l'exclusion des questions épistémologiques de sa résolution. Le second concerne l'occasion manquée d'introduire un nouveau style de raisonnement statistique inférentiel et le renforcement conséquent d'un modèle descriptif particulier. Les deux traits peuvent 
être liés au monopole de l'Académie des Sciences sur la distribution de la reconnaissance scientifique, et au modèle particulier de la statistique qu'elle récompense à travers le mécanisme du Prix Montyon. Tout au long du XIX siècle, l'Académie réaffirmera cette séparation nette entre les mathématiques et les statistiques en même temps que sa définition de la statistique comme un savoir localisé du recueil des données.

\section{La Société de Statistique de Londres, en 1844}

Cinq ans plus tard, de l'autre côté de la Manche, l'utilisation des tables de mortalité suscite un second débat, mais avec des considérations et des modes d'articulation très différents. Un article d'Edwin Chadwick distribué lors d'une réunion de la Société de Statistique de Londres le déclenche. Chadwick était un disciple du théoricien utilitariste Jeremy Bentham, alors l'une des figures dominantes du mouvement de santé publique. Auteur du très acclamé "Rapport sur la condition sanitaire de la population des travailleurs en Angleterre ", il a été mis à contribution pour l'élaboration de la nouvelle loi sur les pauvres (1834).

6 L'article s'intéresse aux aspects statistiques de la réforme, et plus précisément, à la mesure adéquate de la durée de vie, présentée comme une mesure de la santé de la population. Il soulève un débat sérieux au cours duquel l'actuaire ${ }^{20} \mathrm{~F}$. G. P. Neison défie les arguments de Chadwick sur des bases techniques. Les deux articles sont publiés à la suite l'un de l'autre dans le Journal de la Société de Statistique de Londres en avril 1844 ; ils sont suivis par un second article de Neison en décembre 1845, qui développe sa proposition d'alternative technique ${ }^{21}$.

7 La controverse se déroule ainsi en public, et dans une revue associée à une société savante privée, dépendante d'un large public cultivé. Alors qu'en France l'Académie des Sciences arbitrait le débat, en Angleterre ce sont les membres de la Société de Statistique et leurs différents publics qui jouent ce rôle.

\section{Le débat}

\section{Les questions techniques}

L'examen des échanges fait apparaître une très grande variété de types de considérations. Comme dans la controverse Pearson/Yule, qui aura lieu soixante ans plus tard, la raison explicite de la controverse porte sur le choix entre deux mesures statistiques alternatives. En 1844, la question concerne le choix de la mesure de ce que Chadwick considère comme la mortalité de « fait » et ses causes.

La raison explicite de l'article de Chadwick est de promouvoir l'utilisation de l'âge moyen au décès, plutôt que le taux brut de mortalité. Le taux brut serait seulement utile dans le cas exceptionnel d'une population stationnaire, où le rapport nombre de décès/population totale serait équivalent à la moyenne arithmétique des âges au décès. Dans la situation actuelle d'une population croissante, le taux brut sous-estime la mortalité, accroît donc artificiellement les "chances de vie », et sous-évalue ainsi le besoin d'une législation sociale.

Neison défend la construction de tables de mortalité par district et classe sociale. L'âge moyen, explique-t-il, varie avec la structure de la population. Pour illustrer cet effet, il 
procède à plusieurs démonstrations statistiques. Il choisit deux districts pour lesquels les âges moyens au décès et les structures par âge sont différents, et il recalcule l'âge moyen de l'un avec la structure par âge de l'autre, et inversement. La nature de l'exercice est clairement illustrée à la fois par les nombreuses tables qu'il obtient et par son usage étendu du style conditionnel. À la différence de Moreau de Jonnès et de Chadwick, dont les discussions sont toujours exprimées au présent, l'article de Neison est formulé au conditionnel, selon le "nombre de décès qui se seraient produits à chaque période, selon le taux actuel de mortalité des différents districts, si la population avait été la même que celle de Bethnal Green, également selon le nombre d'années qu'ils auraient vécues, et le total de celles-ci » (1844, p. 45). L'essentiel est de démontrer comment «des applications pratiques conduisent à des résultats contradictoires".

Chadwick en revanche rejette de telles préoccupations induisant des confusions entre cause et effet. Selon lui la proportion des enfants et des personnes âgées dans la population résulte du taux de mortalité. Il proteste donc contre les analyses qui traitent la structure par âge comme un facteur indépendant. Quant aux tables de mortalité, il les rejette comme des abstractions générales séparées de l'expérience réelle de populations spécifiques. Il soutient au contraire «que l'expérience réelle (concrète) d'une classe, d'un lieu, d'une période [...] est un guide plus sûr que n'importe quelle table d'assurance déduite de l'expérience d'un autre peuple vivant à une autre période, dans un autre lieu, ou que n'importe quelle loi supposée » (1844, p. 8).

La différence entre les positions de Neison et de Chadwick peut, en partie, être rapportée à leurs différentes approches conceptuelles de la population et de la mortalité comme objets d'enquête. Pour Chadwick, celles-ci sont des constructions empiriques, formées d'individus. Cette position ressort clairement de son emploi fréquent du terme "réel» (actual) pour décrire les taux de mortalité aussi bien que leurs causes, ainsi que de sa critique continue du « raisonnement hypothétique ». Ainsi, insiste-t-il, « c'est seulement par des examens au cas par cas, et sur place, que les faits d'où les principes sont déduits peuvent être correctement distingués ». Pour Neison, au contraire, la mortalité est une force, une loi qui peut seulement être identifiée si elle est abstraite de la variété des autres éléments en jeu dans la production de la population observée.

\section{Les enjeux théorique et pratique}

43 Si Chadwick rejette explicitement l'existence de lois de population «applicables à tous les pays ou à toutes les classes, ou encore à toutes les périodes » $(1844$, p. 8$)$, Neison, au contraire conçoit explicitement les statistiques du mouvement (vital statistics) pour leur utilisation à des fins d'identification des lois et des causes. Alors qu'en France les débats techniques se concentrent sur les types de données, en Angleterre ils portent sur les différentes constructions de la population comme objet statistique et sur le choix des indices. Ces discussions sur le choix de la mesure et sur la population (comme objet d'enquête) sont entrelacées de commentaires sur l'usage auquel chacun destine ces mesures. Pour Chadwick, le but de cette mesure est d'évaluer la salubrité relative des districts locaux et, plus encore, d'établir la responsabilité des conditions environnementales - problème de drainage, d'égout, d'aération, de saleté, d'évacuation des déchets - dans la détermination de ces différences. Comme il l'écrit, «le taux réel de mortalité de la plupart des districts se trouve coïncider principalement avec leur 
environnement physique, et est plus exactement mesuré par les années de vitalité qui ont été vécues, c'est-à-dire l'âge moyen au décès » $(1844$, p. 6$)$.

Les objections de Chadwick aux tables de mortalité et aux discussions en termes de lois sont plus généralement liées à l'usage que les opposants aux réformes de santé publique font de ce langage. Dans sa discussion sur la «fallacy» des arguments malthusiens contre la réforme sociale, il reprend son objection contre toutes formes d'abstraction ou de ce qu'il dénommait «les hypothèses ", dont relève l'usage des tables de mortalité. «Ce que les faits réels font voir de manière frappante est le danger d'adopter des conclusions affectant fortement les intérêts des communautés, sur les raisonnements hypothétiques, et sans une investigation prudente pour savoir s'ils sont soutenus par les faits » (1844, p. 19).

Selon Neison, l'effet de saleté reste encore à établir. Le but des mesures techniques est de fournir des outils pour distinguer entre les effets de causes multiples, y compris la structure par âge, la saleté et la pauvreté, aux différents niveaux de santé publique. Comme Neison l'écrivait en 1845, «il est maintenant juste de supposer que l'emploi ou la profession, la condition de vie ou le rang social, la pauvreté ou la richesse, ont autant d'influence directe sur la durée de vie, que la spécificité d'une localité ou d'une habitation, car l'effet de l'une ou de l'autre des causes supposées influer sur elle n'a pas encore été correctement définies. [...] Afin de déterminer l'influence d'une localité, des classes semblables dans les districts respectifs doivent être comparées » $(1845$, p. 296$)$.

Chadwick comme Neison rend compte des mesures de longévité en tant que moyens d'établir les causes d'une surmortalité. Mais, alors que Chadwick traite les mesures statistiques comme une manière de démontrer l'influence d'une cause particulière (et présumée connue), Neison les conçoit comme un outil de recherche pour distinguer entre les effets de causes multiples. Cet accord sur l'usage général d'un outil et les différences sur son application particulière est semblable à la situation que MacKenzie décrit à propos du débat Pearson/Yule. Là aussi, le choix d'un indice est directement lié à l'usage que les deux scientifiques souhaitent en faire et ces choix, à leur tour, informent le développement théorique ultérieur.

Ces différences peuvent être rapportées à l'engagement des deux hommes dans le mouvement de santé publique, alors divisé entre un courant dit d'ingénieur, influencé par James Bentham et conduit par Chadwick, et un programme médical, soutenu par les professions de santé2 ${ }^{2}$. Alors que les tenants du premier courant recourent à une explication mono-causale de la santé des défavorisés comme résultat des conditions sanitaires, le second appelle à une recherche étendue sur la diffusion et la distribution des différentes maladies. Si Neison n'est pas activement engagé dans la branche médicale du mouvement de santé publique, la plupart de ses amis et collègues le sont, et ses écrits sur la statistique du mouvement appuient explicitement leurs intérêts.

Un an plus tard, Neison publie un long article dans lequel il utilise les tables de mortalité pour comparer la mortalité des différentes classes sociales et des différents districts. Son but est d'explorer les causes de la mortalité élevée en distinguant l'effet de la pauvreté de l'effet de saleté. Le texte se présente comme une alternative claire à l'explication mono-causale de Chadwick.

49 Un examen de l'histoire de la Société de Statistique de Londres et du mouvement de santé publique laisse penser que Neison a gagné le débat au cours des années suivantes. Il appartient au groupe des jeunes membres, incluant notamment William Guy et 
William Farr, qui prennent la direction intellectuelle (et organisationnelle) de la société au début des années 1840 . L'étude des articles publiés au cours des dix années suivantes révèle l'autorité acquise par le modèle de Neison dans les sciences sociales. Alors que la plupart des auteurs n'ont pas ses compétences mathématiques, leurs articles sont parsemés de tables qu'ils ont demandées à Neison.

\section{Modes d'articulation et styles de raisonnement} analyse. La première concerne le degré d'imbrication entre des types différents de considérations, la seconde, le rôle du débat dans la diffusion d'un certain type de savoir-faire statistique. Comme dans le cas de la controverse sur les coefficients de corrélation, les divergences sur le choix des taux sont étroitement liées aux usages propres à chaque auteur de l'outil statistique, ainsi qu'aux différences politiques entre les projets.

51 Chadwick comme Neison se servent des mesures de mortalité et de longévité comme des outils pour établir les causes des variations sanitaires et comme instrument de réforme sociale. Le choix par Chadwick d'un index est directement motivé par son intérêt pour une mesure qui à la fois renseignerait sur l'état de santé des défavorisés et qui lui permettrait de démontrer l'influence des conditions environnementales. Sa négligence des effets de la structure par âge peut être rapportée au fait qu'un tel facteur diminue souvent les taux de mortalité enregistrés, parce qu'il prend en compte les effets d'une population très jeune.

Ce programme politique, à son tour, aide à expliquer son projet de statistique, à savoir l'utilisation des nombres pour fournir une indication générale des domaines d'application de la réforme, plutôt qu'un indicateur déterminé ou un moyen de choisir entre des causes différentes. "Je les défends moins comme des données essentielles, susceptibles d'apporter quelque chose par elles-mêmes, que comme des mesures approximatives, ou des indications de l'étendue de l'opération des causes par des investigations distinctes" $(1844$, p. 8$)$. Son choix d'un index et son engagement dans des discussions techniques peuvent être plus généralement compris comme partie d'une stratégie générale pour promouvoir son approche en tant qu'«ingénieur» auprès du milieu de la santé publique. De manière similaire les positions de Neison sont aussi complètement intégrées à une stratégie. Son engagement technique en faveur des tables de mortalité est directement soutenu par son intérêt à promouvoir la statistique comme instrument scientifique, et par son rôle actif dans la nouvelle direction de la société londonienne de statistique.

Si on se tourne vers les conditions institutionnelles qui peuvent expliquer ce modèle particulier d'articulation, plusieurs observations s'imposent. La première concerne la décision de Chadwick de s'engager dans une discussion des indices statistiques. Il utilisait la statistique dans son travail politique, il n'était pas un statisticien mais un réformateur politique. Le fait qu'il ait choisi d'écrire un article technique et qu'il l'ait présenté devant la Société de Statistique de Londres montre l'étroite relation qui existait alors entre ce que nous considérons comme une organisation scientifique et une organisation politique.

Dans une perspective scientifique, cette situation peut être rapportée au caractère privé des sociétés savantes et de leur dépendance d'un large public. Alors qu'en France 
les dirigeants de l'Académie des Sciences imposèrent avec succès leur modèle épistémologique sur la pratique des statisticiens, en Angleterre leurs homologues de l'Association britannique pour l'Avancement des Sciences échouèrent piteusement. En revanche, les sociétés savantes dépendaient d'un large soutien public. Ces sociétés comprenaient parmi leurs membres aussi bien des hommes politiques, des administrateurs, des réformateurs sociaux que des représentants des élites locales.

Cette situation, à son tour, aide à expliquer l'importance dans ce débat de ce que MacKenzie appelle des « intérêts cognitifs » (des buts pratiques ayant des conséquences théoriques), et plus généralement la dynamique selon laquelle se sont développées les statistiques sociales anglaises. Alors que les partisans de Neison ne pouvaient pas toujours distinguer entre ses considérations techniques et celles de Chadwick, ils pouvaient comprendre les enjeux politiques. Comme je l'ai montré ailleurs, le succès ultime du style de raisonnement de Neison est intimement lié à la victoire de la branche médicale du mouvement de santé publique, et à leur intérêt à produire des statistiques officielles pour satisfaire les exigences techniques du mouvement.

La seconde caractéristique concerne la diffusion d'une forme particulière de savoirfaire statistique. Ce phénomène est lié à la fois au type de considérations techniques en jeu et à l'influence exercée sur le public impliqué dans de telles discussions. Alors que Chadwick et Neison divergent sur la nature des entités statistiques et sur le rôle des statistiques dans l'identification des causes, tous deux utilisent leurs articles pour exercer leurs lecteurs à leur propre utilisation et interprétation des taux de mortalité. La comparaison de Chadwick entre les taux de mortalité générale et l'âge moyen au décès lui sert à expliquer les effets de la croissance de la population (y compris par l'immigration) sur les taux bruts de mortalité et sur l'indépendance de taux en apparence similaires. L'article de Neison conduit cet exposé pédagogique un pas audelà en fournissant des exemples nombreux de la distorsion créée par la structure par âge sur les taux de mortalité, ainsi que l'usage de calculs mathématiques étendus pour extrapoler la population résidante entre deux recensements.

Ces enseignements sont loin d'être confinés aux quelques statisticiens capables de procéder à des calculs mathématiques. Ils sont au contraire diffusés dans un large public d'administrateurs locaux, de médecins et de réformateurs. Pour donner un exemple, le rapport annuel du Registre général qui comportait des exercices similaires, était envoyé à 2000 de ses employés locaux, chargés de recueillir les données du recensement, ainsi qu'à un grand nombre de médecins responsables de l'enregistrement des causes de décès. En outre, ces rapports étaient régulièrement cités par les témoins des Commissions royales d'enquête et dans les rapports locaux, montrant là encore l'ampleur de la diffusion ${ }^{23}$.

\section{Styles de raisonnement, modes d'articulation et conditions institutionnelles}

58 La juxtaposition de l'analyse par MacKenzie de la controverse Pearson/Yule des années 1900-1911 et du débat Chadwick/Neison de 1844 fait ressortir une similarité dans les modes d'articulation entre les différents types de considérations en jeu. Dans ces deux débats, les considérations techniques, pratiques, politiques et idéologiques sont intimement entrelacées. L'insistance avec laquelle Pearson définit les coefficients de corrélation selon l'hypothèse (largement infondée) que les fréquences observées 
reflètent une distribution binomiale, est directement liée à l'utilisation qu'il veut faire de cette mesure, qui à son tour dépend de son programme eugénique. De même, le choix par Chadwick de l'âge moyen au décès comme mesure de la longévité est attaché à la représentation que cette mesure particulière donne de la mortalité, et au soutien qu'elle apporte à son approche d'ingénieur dans la promotion de santé publique. Dans les deux cas les considérations techniques sollicitées sont utilisées pour exprimer des intérêts politiques et vice versa.

Un des traits les plus frappants de ces deux controverses concerne la contribution de ce mode particulier d'articulation à l'avancement du savoir-faire statistique et à l'introduction de nouvelles formes de raisonnement statistique, que ce soit le raisonnement inférentiel dans les années 1840 ou la modélisation mathématique dans les années 1900. Dans les deux cas, une controverse sur des considérations techniques entretenue par des intérêts professionnels et politiques est l'occasion d'une innovation statistique. En 1844 le débat Chadwick/Neison incite Neison à appliquer les tables de mortalité au problème de la discrimination entre des causes multiples de mortalité (insalubrité contre pauvreté). De plus, son exposé soigné et largement diffusé des principes de base du raisonnement statistique inférentiel joue un rôle important dans l'éducation et du même coup dans la diffusion de ce mode de raisonnement. En 1900, la controverse Yule/Pearson permet l'extension des coefficients de corrélation aux variables nominales et de la mathématique aux statistiques.

En France, le débat à l'Académie des Sciences et celui à l'Académie de Médecine en 1867 sur la dépopulation, analysé ailleurs, présentent un schéma très différent ${ }^{24}$. Dans les deux cas, les considérations techniques étaient curieusement séparées des considérations épistémologiques qui, à leur tour, se développent différemment à partir d'éléments politiques et professionnels. En 1838, l'intérêt de Moreau de Jonnès pour les compagnies d'assurance, par exemple, ne joue aucun rôle dans son argumentation à propos de la qualité des données de Demonferrand.

61 L'introduction de considérations épistémologiques pourrait donner à penser que les deux débats, celui de 1838 et celui de 1867, ont joué un rôle décisif dans l'introduction du mode inférentiel du raisonnement statistique. Mais il n'en est rien. En 1838, une confrontation ouverte entre les deux styles de raisonnement est empêchée par l'Académie des Sciences qui intervient en faveur de Demonferrand, mais sur la base d'un modèle statistique descriptif. De manière similaire, une analyse du débat de 1867 montre comment Paul Broca, le principal partisan de l'approche inférentielle, s'appuie sur des arguments descriptifs pour soutenir ses positions politiques et ignorer le potentiel explicatif inhérent aux considérations techniques qu'il a lui-même introduites. Comme en 1838, la discussion de 1867 reste fixée dans le cadre d'un raisonnement de statistique descriptive, dans lequel l'autorité épistémologique des nombres repose sur leur correspondance une à une avec les observations et où les statistiques sont maintenues à l'écart de l'explication.

La juxtaposition de ces deux dimensions des débats statistiques - à savoir le mode d'articulation de considérations différentes au sein des débats et le style de raisonnement statistique en jeu - suggère l'existence d'une relation durable, quoique complexe, entre les deux. À première vue, il n'y a pas de raison pour que les modes d'argumentation et les formes de causalité ou bien les entités statistiques - éléments essentiels d'un style de raisonnement selon la définition de Hacking - soient liés. 
L'analyse menée ici remet toutefois en cause cette idée, suggérant plutôt l'existence de régularités entre les deux dimensions de la controverse statistique.

À un premier niveau, cette association peut être attribuée au contenu des deux styles de raisonnement considérés et, plus précisément, à la place qu'ils réservent aux considérations pratiques. Ainsi, si le style descriptif place une coupure radicale entre un certain savoir et son utilisation, le style inférentiel fait appel à l'emploi des nombres pour « en inférer des généralisations ou décider ce qui doit être fait à la lumière des données et des objectifs ${ }^{25}$ ». Selon cette logique, des styles de raisonnement qui distinguent entre science et politique ne devraient pas recourir à des arguments politiques pour renforcer rhétoriquement des points techniques ; ceux qui font appel à l'emploi des chiffres pour formuler une politique devraient articuler des considérations techniques et politiques à l'intérieur d'une seule discussion. Mais cette explication n'est qu'en partie satisfaisante car les formes descriptives du savoir statistique ont été clairement utilisées à des fins politiques, et il n'y a pas de relation nécessaire entre les usages pratiques et inférentiels du savoir.

Un second type d'explication pourrait découler du Foucault de l'Archéologie du savoir en traitant ensemble le mode d'argumentation et le style de raisonnement comme des parties de la même formation discursive. La difficulté est que cette approche conduit à une sorte de tautologie dans laquelle le mode d'argumentation est redéfini comme un élément du style de raisonnement et où le style de raisonnement explique le mode d'argumentation. Foucault lui-même aurait sans doute recherché l'explication de cette articulation entre les différentes composantes d'un discours statistique particulier (les types d'entité, de propositions, d'explication, et maintenant les modes d'articulation) dans les règles de la formation du discours qui rendent certaines formes possibles, règles qui, comme il l'aurait lui-même affirmé, seraient fondées sur des relations entre des institutions, des processus économiques et sociaux, des modes de comportements, des systèmes de normes, des techniques, des types de classification et des modes de caractérisation.

Je voudrais proposer une troisième explication complémentaire, relative aux mécanismes institutionnels qui informent la dynamique des discussions techniques sur la statistique, afin d'aider à expliquer les différences observées entre la France et l'Angleterre. Cette approche parvient à expliquer non seulement la corrélation qu'on relève entre des modes d'argumentation et des styles de raisonnement, mais aussi la persistance historique des formes descriptives en France et l'introduction des formes inférentielles en Angleterre.

Les deux cas français mettent en évidence l'importance des académies comme espaces quasi officiels permettant la juxtaposition de considérations politiques et scientifiques au sein d'un même débat. En 1838, la médiation de l'Académie des Sciences conduit au déplacement d'un problème épistémologique sur la nature du savoir statistique à un débat plus facile à gérer dans le cadre de la statistique descriptive. En 1867, la critique fondamentale de l'Académie de Médecine contre la méthode numérique fournit à Guérin - le principal opposant à Broca - un argument d'autorité au moment précis où il est le plus vulnérable. Plus généralement, le veto contre l'utilisation des nombres aide à comprendre le malaise de la plupart des participants au débat de l'Académie de Médecine vis-à-vis des arguments techniques de Broca, dépourvus ainsi de force persuasive. 

ministres intéressés par le jugement scientifique des Académies sur des affaires pertinentes au plan politique. En 1838, la question porte sur la qualité scientifique des premières publications du nouveau bureau statistique, en 1867, elle concerne leur évaluation de la gravité de la mortalité infantile et de la pratique de la mise en nourrice. Le fait que, dans les deux cas, les ministres se soient adressés à l'Académie des Sciences et à celle de Médecine, plutôt qu'à des experts-statisticiens membres de sociétés statistiques privées (indépendantes), montre le monopole exercé par les Académies sur les rapports entre l'administration et les activités scientifiques. Dans le cas de la statistique sociale, ce monopole empêche de manière effective la réalisation d'un projet de statistique inférentielle, destiné à identifier, au moyen de l'usage des nombres, des causes et des lois et à influencer une politique sociale, menée par des statisticiens et des réformateurs extérieurs aux Académies.

En Angleterre, en revanche, les débats technico-politiques furent conduits dans des sociétés de statistique privées (indépendantes) face à un public très divers composé de statisticiens, d'hommes politiques, d'industriels, d'hommes d'affaire, de professionnels et de réformateurs sociaux. En 1844, Edwin Chadwick fut le leader reconnu du mouvement de santé publique, alors que Neison était un actuaire en vue. La clôture du débat dépendit finalement, non de l'opinion de juges préétablis, mais plutôt du soutien qu'obtint chaque orateur des membres de la société.

69 Cette différence entre les conditions locales institutionnelles dans lesquelles se déroulèrent les débats peut, à son tour, être utilisée pour relever les différences dans l'accès des «porteurs » des nouveaux modes de raisonnement statistique aux centres du pouvoir intellectuel et de décision politique. En Angleterre, les statisticiens faisaient partie d'un réseau informel liant des élites intellectuelles, sociales, économiques et politiques, qui jouèrent un rôle central dans l'introduction de nouvelles politiques sociales, en particulier dans le domaine de la population et de la santé publique. En termes d'histoire de la statistique, on peut considérer que ce réseau, ainsi que les nombreuses organisations officielles et privées qu'il activait, a procuré une base institutionnelle fondamentale à l'élaboration et à la diffusion des styles de raisonnement inférentiel. En France, en revanche, les statisticiens qui défendaient ces styles inférentiels se trouvaient généralement en dehors des institutions scientifiques regroupant les élites.

Énoncée en termes plus théoriques, cette analyse suggère un modèle explicatif liant conditions institutionnelles, critères de reconnaissance scientifique, modes d'argumentation et styles de raisonnement. Le modèle permet de décrire des différences dans la relation entre la science et l'influence directe des mécanismes par lesquels des controverses sont évaluées, conduites, conclues ou reconduites. Il suggère en outre que ces différences dans la distribution (et le contenu) de la reconnaissance scientifique aident à comprendre des différences dans les formes discursives. Plus précisément les différences du premier type éclairent des différences dans la manière dont des considérations parviennent à être articulées au sein d'une même discussion ; elles éclairent aussi leur poids respectif comme techniques de persuasion. Ainsi, les différences observées dans des lieux institutionnels et discursifs s'avèrent finalement cruciales pour comprendre ses différences nationales dans la chronologie et la diffusion d'un nouveau style de raisonnement. 


\section{NOTES}

1. Voir en particulier T. Porter, The Rise of Statistical Thinking, Princeton, Princeton University Press, 1986 ; L. Krüger, L. Daston et M. Heidelberger, eds, The Probabilistic Revolution, Cambridge, MIT Press, 1987 ; I. Hacking, The Taming of Chance, Cambridge, Cambridge University Press, 1990.

2. I. Hacking, «Statistical language, statistical truth and statistical reason: the selfauthentification of a style of scientific reasoning ", in E. McMullin, ed., The Social Dimensions of Science, Notre Dame, University of Notre Dame Press, 1992, p. 130-157 ; semblable à la notion, plus courante, de "style de pensée ", centrale dans la sociologie allemande du savoir de l'entre-deuxguerres (chez Scheler et Mannheim, par exemple), le «style de raisonnement » met l'accent sur des pratiques discursives, alors que le «style de pensée » accentue la dimension intellectuelle du savoir. Hacking précise ainsi le champ d'étude de Crombie auquel il se réfère dans ses premiers textes: cf. A.C. Crombie, Styles of Scientific Thinking in the European Tradition (3 vol.), Londres, Duckworth and Co, 1994, où l'auteur a rassemblé ses études des vingt années précédentes. La notion de Hacking, issue explicitement de la notion de discours chez Foucault, est aussi comparable à celle développée par L. Fleck dans The Genesis of a Scientific Fact, Chicago, University of Chicago Press, 1981.

3. D. MacKenzie, "Statistical Theory and Social Interests : a case study », Social Studies of Science, 8, 1978, p. 35-83.

4. Alors que le $\mathrm{XIX}{ }^{\mathrm{e}}$ siècle fut marqué par de nombreuses discussions sur la population, souvent avec l'appui de données statistiques, peu de débats portèrent directement sur des considérations techniques, tel que le choix d'un indicateur ou celui des données et de leur contrôle pour la construction d'un taux. Un dépouillement des journaux, des actes des sociétés savantes et des congrès où furent discutées ces questions ne révèle que trois débats de ce type. Ont été dépouillés : les Annales d'Hygiène, le Journal de la Société de Statistique de Paris, les Comptes Rendus des Académies des Sciences, de Médecine, des Sciences Morales et Politiques en France, le Journal of the Statistical Society of London, The Lancet, The British Medical Journal et le Transaction of the British Association for the Advancement of Sciences, Section F en Angleterre.

5. T. Gieryn, «Relativist/Constructivist Programmes in the Sociology of Science: Redundance and Retreat", Social Studies of Science, 12, 1982, p. 279-297, propose une approche critique de ce qu'il appelle l'école "constructiviste/relativiste»; il distingue trois principaux courants : les études, à un niveau macro, du rôle des «intérêts" dans l'établissement des énoncés scientifiques; des études ethnographiques de pratiques de laboratoires pour analyser le caractère local, contingent, de tels énoncés; des analyses de discours qui mettent l'accent sur la façon dont ces énoncés sont formulés. Seul le premier d'entre eux a pris les controverses comme objet privilégié d'analyse. Cf. également D. Pestre, «Pour une histoire sociale et culturelle des sciences. Nouvelles définitions, nouveaux objets, nouvelles pratiques », Annales HSS, L, 1995, p. 487-522.

6. B. Barnes, Scientific Knowledge and Sociological Theory, Londres, Routledge \& Kegan Paul, 1974.

7. Le numéro de 1981 de Social Studies of Science, intitulé « Controversies », constitue la référence essentielle ; les études de cas les plus importantes ont été traduites dans B. Latour, ed., La Science telle qu'elle se fait, Paris, Pandore, 1982 ; cf. également E. Mendelsohn, « The political anatomy of controversy in the sciences", in H. T. Engelhardt et A. L. Caplan, Scientific Controversies. Case Studies in the Resolutions of Closure of Disputes in Science and Technology, Cambridge, Cambridge University Press, 1987, p. 93-124.

8. B. Latour, Science in Action, Cambridge, Harvard University Press, 1987.

9. H. T. Engelhardt, et A. L. Caplan, Scientific Controversies..., op. cit. 
10. S. Woolgar, "Interests and Explanation in the Social Study of Science ", Social Studies of Science, 11, 1981, p. 365-394.

11. A. Pickering, ed., Science as Practice and Culture, Chicago, University of Chicago Press, 1992.

12. Cf. H.-J. Rheinberger et M. Hagner, «Experimental Systems, Objects of Investigation and Modes of Representation", in M. Heidelberger et F. Steinler, eds, Meaning and Varieties of Experiment, Baden Baden, Nomos, 1997.

13. Le Prix Montyon, décerné par l'Académie des Sciences pour les travaux statistiques, était l'un des principaux instruments de reconnaissance scientifique; au cours du siècle, la plupart des statisticiens présentèrent leurs travaux pour l'obtenir. Cf. E. Brian, «Le Prix Montyon de Statistique à l'Académie Royale des Sciences pendant la Restauration ", Revue de Synthèse, IV ${ }^{\mathrm{e}} \mathrm{s}$, II, 1991, p. 207-236.

14. Pour l'ensemble du débat, cf. Comptes Rendus de l'Académie des Sciences, 1, 1835, p. 325 ; 2, 1836, p. 34-36, 437-439 ; 4, 1837, p. 526-527, 698-701 ; 7, 1838, p. 97-99, 248-249, 312-313, 349-354.

15. Directeur de la Statistique Générale de la France récemment créée, Moreau de Jonnès envisageait sa tâche comme la compilation d'une " histoire naturelle ", statistique et exhaustive, de la France. Dans son plan, présenté en 1835, il prévoyait l'organisation des statistiques officielles en quatorze rubriques : territoire, population, agriculture, mines, industrie, commerce, navigation, colonies, administration intérieure, finances, forces armées, marine, justice et instruction publique ; cf. H. Le Bras, «La Statistique générale de la France », in P. Nora, ed., Les lieux de mémoire, Paris, Gallimard, II, 1984, p. 317-353. Sur les différences avec le programme du General Registrar's Office, cf. L. Schweber, The Assertion of Disciplinary Claims in Demography and Vital Statistics : France and England, 1830-1885, Princeton University, thèse de Ph.D, 1996, multig.

16. Comptes Rendus de l'Académie des Sciences, 7, 1838, p. 249, 437.

17. Ibid., p. 354.

18. Merci à Stéphane Baciocchi pour avoir remarqué cette double interprétation.

19. Sur cet ensemble de questions, cf. L. Schweber, The Assertion of Disciplinary Claims..., op. cit.

20. La profession d'«actuaire", spécialiste du calcul financier auprès des compagnies d'assurances, était alors une profession plus spécialisée et mieux reconnue en Angleterre qu'en France [N.d.R.]

21. E. Chadwick, "On the best Modes of representing accurately, by Statistical Returns, the Duration of Life, and the Pressure and Progress of the Causes of Mortality amongst different Classes of the Community, and amongst the Populations of different Districts and Countries ", Quarterly Journal of the Statistical Society of London, 7, 1844, p. 1-40 ; F. G. P. Neison, " On a Method recently proposed for conducting Inquiries into the Comparative Sanitary Condition of various Districts, with Illustrations, derived from numerous places in Great Britain at the period of the last Census ", ibid., p. 40-53, et " Contributions to Vital Statistics, especially designed to elucidate the Rate of Mortality, the Laws of Sickness, and the Influences of Trade and Locality on Health, derived from an extensive Collection of Original Data, supplied by Friendly Societies, and proving their too frequent Instability ", ibid., 8, 1845, p. 290-343 ; 9, 1846, p. 50-76. Sur le déroulement du débat, Londres, Statistical Society, Minutes, 15 décembre $1843 ; 12$ janvier, 16 février, 8 et 15 mars 1844.

22. Cf. R. MacLeod, «The Anatomy of State Medicine : Concept and Application ", in Medicine and Science in the 1860s. Proceedings of the 6th British Congress on the History of Medicine, Londres, Wellcome Institute of the History of Medicine, 1968, p. 199-227.

23. Sur les activités statistiques du General Registrar's, cf. J. Eyler, Victorian Social Medicine, Baltimore, Johns Hopkins University Press, 1979, et S. Szreter, « The GRO and the Public Health Movement. I Britain, 1837-1914 », Social History of Medicine, 4, 1991, p. 435-463.

24. Cf. L. Schweber, The Assertion of Disciplinary Claims..., op. cit. ; les diverses interventions ont été publiées in Bulletin de l'Académie de Médecine, 32, 1866-1867, passim. 
25. I. Hacking, " "Style" for Historians and Philosophers ", Studies in the History and Philosophy of Science, 23, 1992, p. 1-20.

\section{RÉSUMÉS}

La sociologie des sciences traite les controverses comme un objet privilégié pour examiner l'hétérogénéité des considérations à l'œuvre dans la production du savoir scientifique. L'étude des débats sur l'usage des tables de mortalité pour analyser, en France et en Angleterre, les statistiques de population au XIX ${ }^{\mathrm{e}}$ siècle étend l'enquête aux différences entre nations, à propos de la façon dont sont articulés les différents types de considérations. En Angleterre, les différentes considérations - techniques, épistémologiques, politiques, institutionnelles - sont fortement intégrées, alors qu'elles suivent en France des logiques différentes. Cette comparaison suggère également que les controverses peuvent être analysées comme l'un des nombreux types de micro-événements lors de l'introduction (ou du rejet) de nouvelles formes de raisonnement statistique (introduction de formes inférentielles de raisonnement en Angleterre dès 1844, résistance des statisticiens français, en opposition aux mathématiciens, à de telles formes). À partir des relations entre mode d'argumentation et styles de raisonnement, l'article suggère une explication institutionnelle pour des différences nationales à partir d'éléments qui proviennent des controverses elles-mêmes.

The sociology of science tends to treat controversies as a privileged object to document the heterogeneity of considerations at play in the production of scientific knowledge. An examination of 19th century debates over the use of mortality tables in the analysis of population statistics in France and Britain extends this problematic by inquiring into national variations in the way in which different types of considerations are articulated. In England technical, epistemological, political and institutional considerations prove to be highly integrated whereas in France they follow separate logics. This comparison also suggests that controversies can be analysed as one of the many types of micro-events in the introduction (or rejection) of new forms of statistical reasoning (introduction of inferential forms of reasoning in England as early as 1844 and the resistance of French statisticians, in counter-distinction with mathematicians, to similar forms). The article speculates on the relation between modes of argumentation and styles of reasoning and suggests an institutional explanation for national differences based on traces found within the controversies themselves.

\section{AUTEURS}

\section{LIBBY SCHWEBER}

Libby Schweber (Berlin, Max Planck Institut fur Wissenschaftsgeschichte/Harvard University) travaille sur l'émergence des différentes disciplines des sciences sociales. Adoptant une perspective micro comparative, elle se sert de l'histoire de la statistique sociale pour explorer des différences nationales dans la (re)configuration des sciences sociales au cours du XIX ${ }^{\mathrm{e}}$ siècle. Elle achève actuellement un livre sur l'histoire de la démographie et des « vital statistics » en France et en Angleterre. 